\title{
The analysis of multichannel MAC protocols which implement a control channel
}

\author{
Mthulisi Velempini ${ }^{1}$ and Mqhele E. Dlodlo ${ }^{2}$ \\ ${ }^{1}$ North-West University, South Africa, Mmabatho \\ ${ }^{2}$ University of Cape Town, South Africa, Cape Town
}

\begin{abstract}
The three-way need for higher data rates, good quality of service and ubiquity in a converged all IP communication cloud drives research in wireless communication. Wireless access networks are envisaged candidates of the next generation wireless networks. The various access networks will be integrated with other technologies including the wired backbone. The major issues in an all IP and converged networks are: quality of service, seamless handover and network capacity. Emerging research seeks to address these open research issues; for example the implementation of multi-channel and multi radio MAC protocols in $W M N$. In this paper we analyze and evaluate the effectiveness of multi-channel and multi radio techniques in WMN. The shortcomings of these schemes are highlighted and possible solutions are suggested. The signalling delay metric is used for evaluation purposes. The focus is on the performance of the control channel identified as the critical performance metric of multi-channel MAC protocols.
\end{abstract}

\section{KEYWORDS}

Broadcasting; Control channel; Data Structures; Multi-channel; Signalling delay

\section{INTRODUCTION}

The need for high speed and broadband networks has prompted researchers to consider wireless mesh networks (WMN) as a possible candidate for the next generation wireless networks (NGWN). The multichannel and multi radio techniques are considered in WMN implementation as possible strategies for achieving the anticipated higher data rates. The next generation of access networks is envisioned to offer high data rates and good quality of service (QoS). However, WMN does not meet the requirements of the next generation networks (NGN). For example, the MAC protocols are not scalable and are not optimized for multiple channels.

The IEEE 802.11 family of standards which was designed for single channel is considered for WMN. In recent years significant work has been done in designing MAC protocols which are suitable for multichannels and multi radio networks. In this paper, we review multi-channel schemes which implement either a temporal or a dedicated control channel. The shortcomings of these schemes are discussed and possible solutions are suggested. The paper also discusses the open research issues.

The performance of a number of schemes are evaluated in terms their ability to either reduce or increase the signalling overhead of the control channel. We attest that the success and the effectiveness of multi-channel MAC protocols depend on the reduction of the signalling payload of either the control channel or the control window. Furthermore, the schemes have to be cost effective and less complex. We also evaluate these schemes in terms of their ability to deliver broadcast packets and the amount of processing done by a mobile node. In addition, the multi-channel MAC schemes should ensure total connectivity of the network. 
In this paper, multi-channel MAC protocols are classified into the following five taxonomies: windows based, dedicated control channel, contention based, user defined and channel hopping multi-channel MAC categories. The windows based protocols split a communication phase into control and data windows. In these schemes, data channels are reserved during the control window through a control channel. However, there are a number of strategies of implementing a control channel.

The multi radio schemes employ two radios, a control channel radio and a data channels radio. The data channels radio is either user defined or contention based. The data channels radio switches between data channels or stays on a user defined channel for a set duration. The control radio transmits control packets while the data channel radio transmits data and acknowledgement (ACK) packets.

The dedicated control schemes implement a single transceiver which switches dynamically between all the available channels. Data channels are reserved through the control channel; thereafter the radios have to switch onto the reserved data channels to transmit data packets.

The rest of the paper is organised as follows: the classification of multi-channel MAC schemes is presented in Section II. The contention based multi-channel multi radio techniques are presented in Section III. In Section IV, we discuss the user defined multi-channel multi radio techniques. In Section V, temporary control channel MAC protocols are discussed. Section VI presents dedicated control channel MAC protocols. Section VII briefly discusses the channel hopping MAC schemes. Open research issues are discussed in Section VIII. Section IX concludes the paper.

\section{Classification of multi-Channel MAC protocols}

A number of multi-channel MAC protocols classifications have been suggested. They can be classified according to their function, design or main objectives. In this Section, we classify the multi-channel MAC protocols according to the number of radios employed and how data channels are reserved. The common aspect of these protocols is the implementation of a control channel either in a temporary or dedicated form. There are many other multi-channel MAC protocols of interest which do not implement the idea of a control channel. Some of these protocols will be discussed briefly under the channel hopping category. The focus of this paper is on the implementation of the control channel and its effect on the performance of multi-channel MAC protocols.

Fig. 1 depicts the five main classifications of Multi-channel MAC protocols. These are the temporary control channel, the dedicated control channel, the contention based, the user defined and the channel hopping categories. With these classifications, this paper seeks to evaluate the impact of the signalling overhead on the capacity of the control channel.

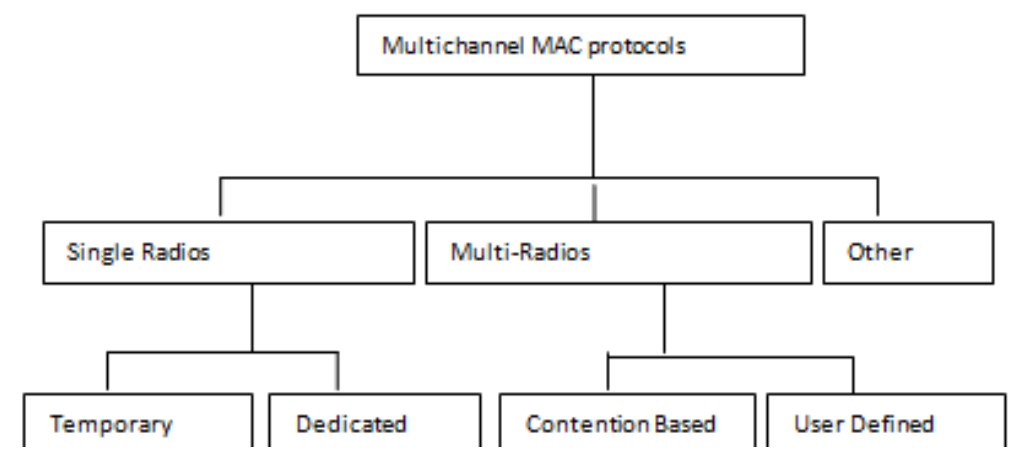

Figure 1. The classification of Multi-channel MAC protocols.

The temporary control channel MAC protocols employ one channel as a signalling channel during data channel reservation during the control window phase. During the data window phase, the control channel is 
used as a data channel. This is in contrast to MAC protocols which employ a dedicated control channel. The dedicated channel is only used signalling purposes. Lastly, the multi radio MAC protocols can be classified either as user defined or contention based.

Multi radio MAC protocols employ a notion of a control channel; however, they differ in the selection and coordination of data channels. The user defined protocols assign a data channel to a specific terminal; while the contention based protocols all the data channels are accessible to all the available terminals. The terminals contend for data channels when they have data frames to transmit. A data channel is assigned to a sender/receiver to transmit data and the assigned nodes have control of the data channel only during data transmission. After the transmission of data packets, the data channel is released and assigned to the next pair. Lastly, in the fifth category, channel hopping multi-channel MAC protocols such as the split phase, and channel hopping algorithms are evaluated.

\section{CONTENTION BASED MULTI-CHANNEL MULTI RADIO TECHNIQUES}

In this Section, we evaluate the multi-channel MAC protocols which employ two radios. One radio is tuned on the control channel and the other radio switches between the data channels. The terminals do contend for the data channels and reserve them through the control channel with the aid of a data structure. The data structure keeps track of the busy channels and lists all the idle channels to assist terminals in reserving data channels.

In [1], a multi-channel MAC protocol called the Dynamic Channel Assignment (DCA) is proposed. The paper is one of the first publications in the area of multi-channel MAC protocols. The DCA scheme implements two radios, one for the control channel and the other for data channels. There is one control channel and $n$ number of data channels. The control channel is employed for signalling purposes. Nodes contend for the control channel using the Carrier Sensing Multiple Access with Collision Avoidance (CSMA/CA) protocol to reserve one of the available data channels. The control packets, Request to Send (RTS), Clear to Send (CTS) and the Reserve (RES) are transmitted on the control channel. The data frames and the ACK packets are transmitted on the available data channels. The RES is a new control packet which increases the signalling overhead by at least $47 \%$.

The data channels are assumed to be of equal bandwidth, and that the second transceiver can switch dynamically between data channels. Unfortunately, in a multi-channel environment

transceivers do incur a switching cost of up to $224 \mu \mathrm{s}$ [2], [3]. If a transceiver switches regularly, the switching cost severely degrades the performance of the protocol.

Data channels are reserved through a data structure called the channel usage list (CUL). Each node is expected to maintain and update its CUL every time it overhears a control packet. The RTS packet includes the free channel list (FUL), a list of channels which are available for use in the sender's communication zone. Upon receiving the RTS packet, the receiver has to check its CUL against the sender's FUL. If there is a common data channel available at both sender and receiver, the receiver selects the free data channel and sends a CTS packet to the receiver. The receiver then sends a RES packet to reserve the selected data channel.

The RES packet is an additional control packet which was first introduced in this protocol. The introduction of the RES packet increases the signalling delay by at least $47 \%$ which degrade the performance and capacity of the control channel. Furthermore, the RES packet fails to inhibit hidden terminals which are in the communication range of the receiver.

The proposed DCA does not provide a solution to a scenario where a common free channel is not available. It assumes that a free channel is always available on both the sender and receiving nodes' communication zones. The protocol is complex and too expensive in terms of hardware cost. The use of the two radios increases the hardware cost of mobile devices. Lastly, a mobile device with two radios suffers from signal linkages, where signals from one radio interferes the signal of the other radio. 
The bandwidth of data channels is underutilized during the reservation phase. The data channels lie idle and their bandwidth is wasted. The data channels are subjected to a multi-channel scheduling cost (MSC) in which data channels lie idle in each phase waiting for their turns to transmit data. The control channel cannot schedule data transmissions to all the data channels simultaneously. Some data channels have to wait for longer durations before they are reserved. We refer to the total underutilized bandwidth of all the data channels as the MSC. Unfortunately, the MSC is repetitive and significant.

A similar protocol is proposed in [4]. The protocol is referred to as DCA with power control. It is designed to solve the following: the channel assignment, medium access and the power control challenges. Data channels are reserved through the exchange of RTS/CTS/RES control packets with the aid of the channel usage list (CUL) and the free channel list (FCL) data structures. The setup and functionality of the protocol is similar to [1]. It also suffers from the longer control channel signalling delays, the signal leakage problem, complexity and high hardware costs. The capacity and the performance of the multi-channel MAC protocols can be improved by reducing the control channel signalling delays and overhead costs.

A kernel based scheme is proposed for multi-channel systems with multi-interfaces in [5]. The interfaces are fewer than the channels; hence the interfaces have to switch between the available channels. In the proposed scheme, the interfaces incur a switching penalty of $5 \mathrm{~ms}$. Interestingly, frequent interface switching degrades significantly the performance of the protocol. A new channel abstraction module was therefore added to offer a virtual switching mechanism. The channel abstraction module resides between the network layer and the interface device drivers. The channel abstraction module was designed to reduce the amount of the interface switching penalty.

Nodes are equipped with two interfaces, one fixed and the other switchable. Nodes are configured to select different fixed channels, which are advertised using broadcast hello messages to inform other nodes. Nodes select fixed channels on temporary basis. The frequency of channel selection and the flooding of the network with Hello messages is a design challenge.

The use of broadcast hello messages to advertise node's fixed channel wastes bandwidth. The broadcast packets should be sent on all the channels to reach all the nodes. Some nodes do not receive broadcast packages due to the missing receiver problem and the logical portioning of the network given user preferred fixed channels, furthermore they increase network traffic significantly, congesting it.

Nodes keep a unicast table to record the fixed channels of their neighbours. Given the fact that, mobile nodes have a limited processing power and storage capacity, this may impact negatively on their capacity and functionality.

In [6] and [7], a protocol similar to [5] is proposed. As such, the same limitations were also observed.

The paper in [8] proposed a multi radio multi-channel scheme. A single channel is set aside as a control channel and the rest of the channels are earmarked for data transmission. The protocol uses a data structure called a channel list (CL) which is used for data channel reservation. The scheme suffers from the signal leakage problem caused by radios transmitting close to each other. Nodes are also allowed to reserve channels which are currently in use. Nodes defer their transmissions on in use data channels until they are free. Nodes processing overhead is too high, a challenge for mobile nodes which have limited processing and storage capacity.

The scheme proposed in [9] also implements a multi interfaces and multi channels technique. It assumes that a node can send and receive on a call by call basis. The control interface/channel is designed for the transmission of broadcast HELLO packets and for routing purposes, while the following: RTS/CTS/DATA/ACK packets are transmitted on the data interface.

A number of multiple channel and multiple radio techniques assume that broadcast packets can be delivered to all the available nodes simultaneously. However, this may be possible given the MRP. This fact was noted in [10] where broadcasting and broadcast latency was analyzed in multi-channel and multiradio schemes. The paper analyzed the broadcast latency in multi radio multi-channel networks and noted that it is a design challenge caused by channel selection algorithms and nodes communicating on different channels - MRP. 
The proposed protocol in [11] employs two network interfaces and is designed to reduce the channel negotiation cost. The proposed scheme is called the Connection-Oriented Multi-channel MAC (COMMAC) protocol. It divides the available bandwidth into one control channel and $\mathrm{n}$ data channels. The channels are assumed to be of equal bandwidth and that they are non-overlapping which is not the case. The control packets, RTS, CTS, and RES are transmitted on the control channel including the broadcast packets. The data frames and ACK packets are transmitted on the data channels.

The nodes are equipped with two radios, a control radio and a data radio. The control radio is tuned on the control channel while the data radio switches between selected data channels. Nodes maintain two tables, a Neighbour Status Table (NST) and a Channel Status Table (CST). A given node records all the channels used by its neighbours in its NST, as well as the control packets overhead. It then records the Network Allocation Vectors (NAV) of data channels in the CST. The information required to update both the NST and the CST is contained in the RTS, CTS and RES control packets.

The protocol suffers from signal leakages owing to two network interfaces placed close to each other. Furthermore, it is degraded by high switching and signalling overhead. It is an expertise and complex scheme.

The proposed RTS is 24 bytes long, 4 bytes longer than the current RTS packets. The proposed CTS packet is also longer than the conventional one by one byte and is now 15 bytes long. The protocol also makes use of a new packet called the RES packet which is 16 bytes long. The new RES packet together with proposed RTS and CTS packets do degrade the bandwidth of the control channel. They also increase the channel negotiation cost and the control channel overhead costs by at least $62 \%$ due to longer transmission durations of the three packets.

Despite the use of two network interfaces, the scheme also suffers from the MSC which is repetitive. Furthermore, data channel network interfaces are subjected to multi-channel switching delays.

The protocol does not consider the multi-channel switching penalty and the timeouts of the control packets are set to the end of the ACK transmission. This worsens the effect of the MSC. The timeouts may be reconfigured and optimized taking into consideration the fact that the control and the data channels use two different network interfaces. The readjustment of the timeouts will reduce the effects of the MSC and the signalling overhead.

The scheme in [12] employs multiple interfaces, a control channel and data channels. The common channel is used for channel assignment. The other channels called the traffic channels transmit data and ACK packets. The first radio is fixed on the common channel while the second one switches dynamically between the traffic channels. Nodes maintain traffic channels usage table which is updated using information overheard on the common channel. The common channel can also be used as a traffic channel when its traffic is low. On the other hand, if a node is aware of the destination's traffic channel, the control handshake is performed on the given traffic channel. These packets are not heard by other nodes, as a result other nodes fail to update their traffic channel usage tables. Nodes use information overheard on the common channel to update their tables.

The protocol introduces six new control packets which increase significantly the payload of the control channel. The additional packets are the Negative clear to send (NCTS), Request to find/acknowledgement to find (RTF/ATF), Request to change traffic channel/acknowledgement to change traffic channel (RCT/ACT), and NAV broadcast (NBC). The scheme is also complex and expensive in terms of hardware costs due to the use of two radios. The MSC is too high due to high signalling cost which has increased more than $100 \%$ owing to too many control packets. The control packets take up a substantial amount of bandwidth, and as a result the utilization of traffic channels is low and ineffective.

The traffic channel radio listens on one traffic channel until it has data to send on a different traffic channel or when the current traffic channel becomes congested. However, a change in traffic channel should be advertised on the common channel through broadcast packets. The use of broadcast packets increases the overhead costs and the complexity of the protocol. Delays may be incurred while a node contends for the common channel to advertise its intention to change its traffic channel. The broadcast packets may not reach all the nodes due to the MRP. 
When the nodes have full knowledge of each other's traffic channels, the common channel and the common channel radio are underutilized since nodes will use the traffic channel radio to communicate directly with their destination nodes on given traffic channels. Joining nodes also fail to acquire neighbour information to update their tables.

In the event of NBC messages being destroyed due to packet collisions, the protocol becomes highly unstable and communication may be impossible. Nodes' neighbour tables are out dated and packets are sent on wrong traffic channels resulting in high incidences of node deafness problems. The scheme also employs a channel assignment and channel reassignment algorithm which increases the payload of the protocol and introduces more signalling delay related costs.

The main challenge of the multi radio systems is cost and complexity. They waste bandwidth on both the control and data channels. They also suffer from the MSC which is repetitive. On the other hand, when the control channel is busy, data channels would be idle waiting to be utilized. After the pair has reserved a data channel, the data channel radio may have to switch onto the reserved data channel increasing the MSC. For example, Fig. 2 shows how the multi-radio systems under-utilize both the control and the data channels.

The top row in Fig. 2 depicts the control channel. The middle and the bottom rows represent any two data channels. Two data channels were chosen for simplicity otherwise more data channels can be considered. As shown in the figure all the channels have busy and idle durations. Nodes first exchange the control packets on the control channel to reserve the available data channels. The nodes then switch onto the reserved data channels while the control channel remains idle. The data channels lie idle during the exchange of RTS/CTS packets on the control channel. The nodes incur two channel switching delays, when they switch from the control channel onto the data channels and when they switch back onto the control channel. However, the switching durations were not highlighted in the diagram. The two channel switching delays increase the idle durations of both the control channel and the data channels.

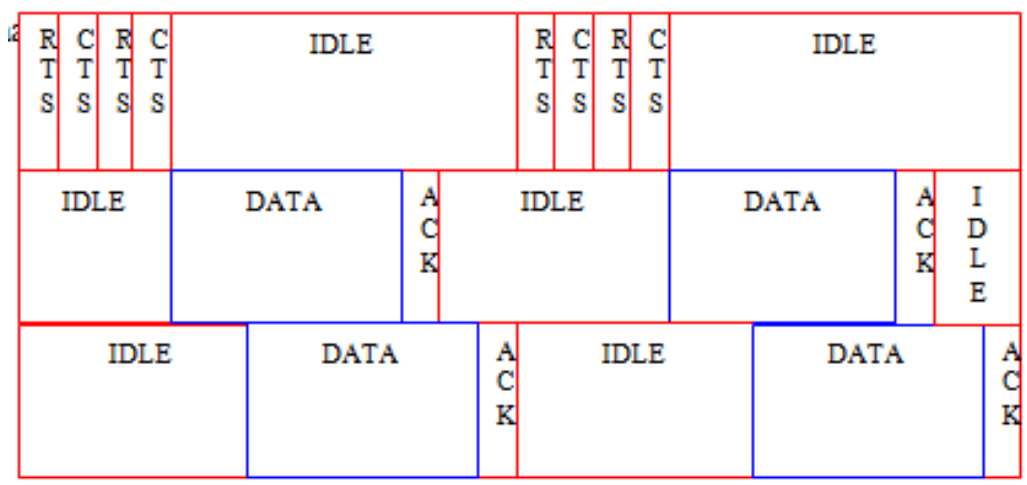

Figure 2. The impact of multi-transceivers on the utilization of channels

Again, in Fig. 2, it can be seen that these idle slots are recurring after every cycle. A significant amount of bandwidth is therefore wasted due to these repetitive idle slots on all the channels. This is a scheduling challenge caused by the implementation a common control channel.

The data channels are reserved when they are free and when the current nodes have switched back onto the control channel. The timeouts are designed such that all nodes overhearing the control packets should set their NAV values to expire at the end of the transmission of ACK packets. Unfortunately, data transmission in multi-channel networks includes two channel switching delays. Hence there is a need for a proactive mechanism for the reservation of data channels which reduces the MSC. Two possible solutions are to reserve a data channel before the current transmission is completed, or to reduce the amount of control channel signalling overhead. The proposed techniques can be combined to reduce or eliminate the MSC.

Figure 3 illustrates how data channels are underutilized during the data channels reservation window. When the RTS and CTS packets are exchanged in the control channel to reserve the available data channels, the 
bandwidth of these data channels is wasted. Interestingly, the wasted of the bandwidth is recurring and very significant. On the other hand, when communication switches onto the data channels, the control channel lies idle. This means that all the available channels are underutilized. This multichannel scheduling challenge therefore requires urgent attention for efficient utilization of available channels.

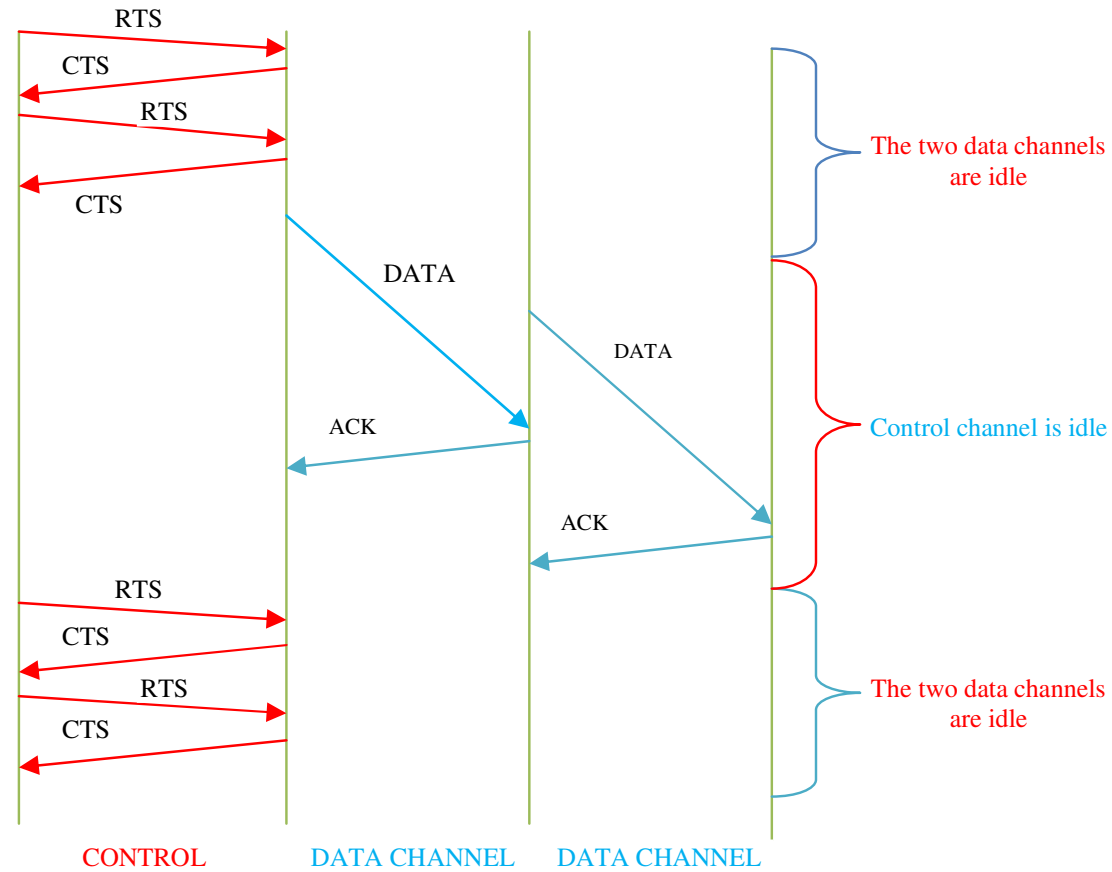

Figure 3. The Underutilization of the Control and Data Channels.

The MSC requires a good, and effective scheduling and data channel coordination strategy. An effective MSC solution may release more bandwidth which may improve the scalability of multi-channel MAC protocols.

\section{USER DEFINED MULTI-CHANNEL MULTI RADIO TECHNIQUE}

Multi radio multi-channel MAC protocols which allow nodes to select home data channels are classified as user defined. The nodes select fixed data channels and broadcast messages to notify other nodes about their selected data channels. In the previous section, nodes do contend for any available data channels and thereafter release them at the end of data transmission. In contrast, the user defined multi-channel multi radio protocols retain selected data channels after data transmission. The selected data channels are used as home data channels until a node selects a different fixed data channel.

In this Section, the effectiveness of user defined multi-channel multi radio MAC protocols in reducing the signalling overhead cost of the control channel is evaluated. The utilization of both the control and the data channels is also analyzed. The Section concludes with the analyses of the general shortcomings of user defined multi-channel multi-radio MAC protocols. The possible solutions and research directions are also discussed.

In [13], a multi-channel and multi-interface MAC protocol is proposed. The scheme proposes the use of multiple interfaces in combination with channel assignment and routing techniques. The scheme is designed to use multiple channels and multiple interfaces effectively. Unfortunately, it does not ensure total network connectivity and it partitions the network into several segments. Nodes listen on different channels and tune on different interfaces thereby segmenting the network logically. Furthermore, it is not feasible to 
have many interfaces on a single mobile device. Devices with multi-interfaces also suffer from signal leakages.

A hybrid channel assignment scheme which does not require clock synchronization is proposed in [14], [15] and [17]. The protocol employs one fixed interface and one switchable interface. The proposed protocols integrate both the static and the dynamic channel assignment strategies into one strategy called the hybrid approach. The static approach is employed to solve the rendezvous problem and to ensure that nodes can synchronize. The dynamic approach is employed to increase the utilization of channels. The objective of the dynamic approach is to facilitate the effective use of multiple channels.

The schemes also implement multi queues, a queue for each channel. The switchable interface has to stay tuned on one channel for a set period to transmit packets for a given data channel queue. The implementation of the multi queues requires more processing power and disk space, a limitation of mobile nodes. Furthermore, the scheme introduces unfairness as it transmits packets for one queue for a set period of time while the other queues are not processed. It is not sensitive to delay bounded packets. Data frames in the next queue are only transmitted after the expiry of the transmission timeslot of the current queue which degrades the performance of the protocol.

The introduction of Dynamic staying Time (DST) and Fixed Staying Time (FST) though novel, requires a lot of processing and knowledge of the length queues which degrades the performance of the protocol. The dynamic waiting time also requires processing time to set a value for a given channel. The functionality of the protocol also depends on the collision probability unfortunately, this probability is estimated, at the actual values are not used.

The user defined multi-channel multi radio MAC protocols are similar to contention based multi-channel multi radio MAC protocols. They differ in the selection and release of data channels. In contention based protocols, nodes reserve a data channel through the control channel with the aid of the data structures. The nodes then switch onto the reserved before transmitting the data packets. The data channel is released after data transmission.

The user defined class of MAC protocols retains the data channel after data transmission and nodes do not have to switch onto reserved data channel. The data channel radios therefore do not switch between channels during data transmission. They only switch when a node selects a new fixed data channel after a set period of time. Hence there is no frequent channel switching and the effects of the MSC are minimized. However, the MRP and the connectivity of the network are of concern.

In Fig.4, the top row depicts the control channel. The bottom two rows represent the two data channels. The data channels lie idle, while reservation takes place on the control channel. While data is transmitted, the control channel remains idle. The idle slots degrade the capacity of the control and data channels. However, the implementation of user defined data channels reduces the MSC on channels.

The multi-channel multi radio protocols waste bandwidth on both the control and data channels. Their effectiveness in the utilization of the control and the data channels does not justify the use of multiple radios. There is need for a trade off between the cost of hardware and channel utilization which may improve the capacity of the control channel and the performance of the network.

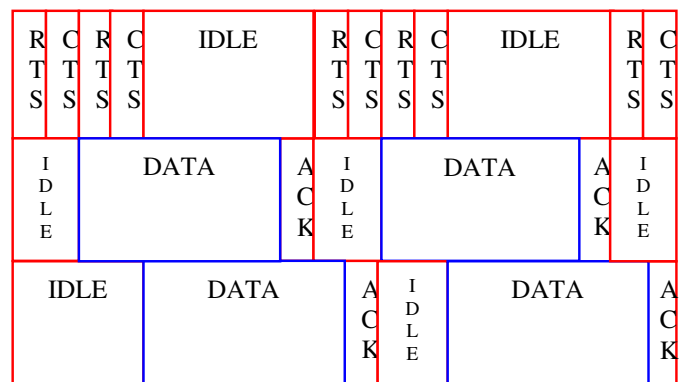

Figure 4. The impact of the Multi-channel Scheduling Cost in user defined multi-channel multi radio MAC protocols. 
The design goals of MAC protocols should not be centred on increased end to end throughput at the expense of efficiency. The efficiency of the protocol should not be sacrificed for expensive techniques such as multiple radios schemes. There is need for optimal mapping of the number of channels onto radios. The optimal number of channels that can be supported effectively by a single radio may be established. Such an optimal mapping may assist MAC designers in designing more scalable and more effective multi-channel multi-radio MAC protocols.

The MSC can be addressed as illustrated by Figure 5. In this case, instead of waiting for all the data channels to be reserved before switching onto the data channels, one can switch to any data channel immediately after it has been reserved. In this way, the idleness of the data channels may be reduced. However, the strategy does not address the challenges of the MSC and the underutilization of the control remains unsolved.

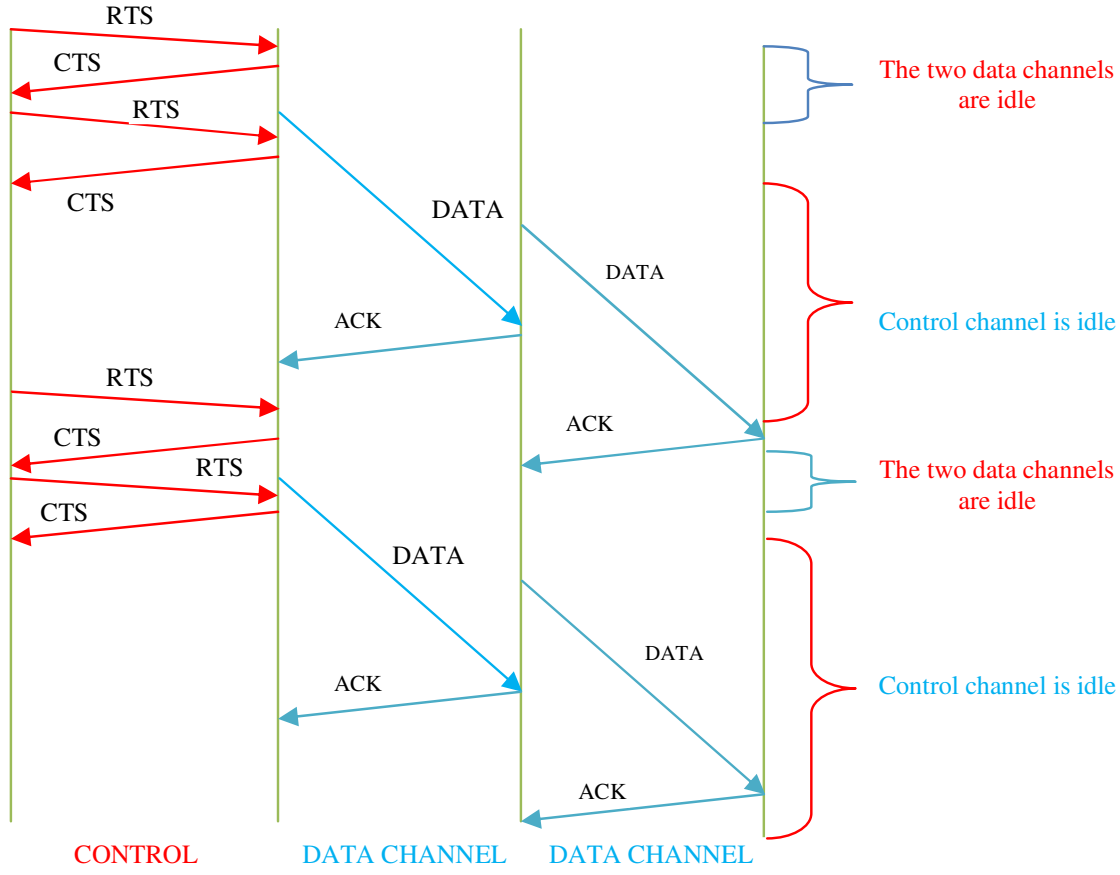

Figure 5.An illustration of how control and data windows waste bandwidth

\section{TEMPORARY CONTROL CHANNEL MULTI-CHANNEL MAC PROTOCOLS}

In this Section, multi-channel MAC protocols which employ a single radio are presented and evaluated. The emphasis is on MAC protocols which set aside one channel for signalling purposes. However, the signalling channel can also be used as a data channel. The communication process is divided into two windows, the control and the data window. The control channel is used as a signalling channel inside the control window and thereafter as a data channel during the data window. During the control window data channels are reserved. Once the control window has expired, nodes start transmitting data frames on the reserved data channels in the data window.

The detailed descriptions of these protocols are discussed in the sequel. The shortcomings and the strengths of these protocols are reviewed. The emphasis is on how the design and the functionality of these protocols affect the capacity of the control channel, which is considered as the driver of multi-channel MAC protocols.

The Extended Receiver Directed Transmission protocol (xRDT) in [16] is one of the multi-channel MAC protocols which implement a temporary control channel. The xRDT employs a temporary control channel 
called the quiescent channel. The protocol uses a dedicated busy tone channel to coordinate the access to data channels. The scheme requires an additional radio tuned on the dedicated busy tone channel. A node which wants to use a given data channel has to first sense the corresponding busy tone channel for busy tone signals. If the busy tone channel is sensed busy, the node has to defer its transmission, but if it is free, the node contends for the data channel.

Each node in the xRDT protocol should select a quiescent channel to listen on. The quiescent channels serve as the home channels. Nodes switch from quiescent channels onto reserved data channels to transmit data. They then return onto their quiescent channels after data transmission. They also broadcast Data Transmission Messages (DTM) upon their return onto their quiescent channels to invite other nodes which have been waiting for their return. The returning node has to invite other nodes to initiate communication with it. The DTM is an invitation message which is sent to other nodes which have been waiting for the return of a particular node to its quiescent channel, to initiate communication with it. Every node upon its return anticipates that there are nodes which are waiting to communicate with it. As a result, nodes flood the network with DTM which degrades significantly the performance of the network. A lot of bandwidth is also wasted when the network is flooded with the DTMs.

The reservation mechanism of this protocol has some shortcomings. The scheme allows the receiver to reserve a data channel in the process notifying only the nodes which are in the receiver's communication zone. The nodes which are hidden to the receiver are not inhibited. These hidden nodes interfere with data reception destroying data packets and prompting nodes to retransmit these packets which further degrades the performance of the protocol.

The selection of the quiescent channels is done periodically. It is based on the load of data channels. A data channel with the least load is selected. Communication with a node takes place on its quiescent channel. The xRDT protocol sends busy tones signals in place of the CTS and the ACK packets. Furthermore, broadcasted packets cannot reach all the nodes which are listening on different quiescent channels.

The paper in [16] also proposed another protocol called the Local Coordination-based Multichannel MAC (LCM MAC). The LCM MAC was designed to address the shortcomings of the xRDT. Control packets are sent on a common channel during the control window while data packets are sent on all the channels during the data window. The control channel is used as a data channel inside the data window to transmit data. The common channel is employed as a signalling channel and is known as the default channel during the control window.

Data channels are reserved during the control window therefore, nodes switch to the data window to transmit data packets. In the control window only one channel is utilized, the default channel. The data channels lie idle and their bandwidth is wasted. More bandwidth is also wasted when the nodes switch on the data channels. Nodes take turns to transmit on the reserved data channels during the data window. This means that some nodes have to wait for longer periods during the data window for their turns to transmit data. The protocol allows more than one pair to reserve one data channel during the control window. The nodes then scheduled to take turns to transmit data during the data window.

The LCM MAC first selects a master node to coordinate the reservation of data channels. The master node first advertises a control and data window schedules thorough the RTS. The RTS length has been increased to accommodate the additional fields for control and data window durations. The additional fields do degrade the performance of the scheme. The RTS also stores the list of all data channels which are free at the sender. When the receiving node receives the RTS packet, it replies with a CTS packet and selects the channel Identification (ID) for the channel which is free at both the receiver and sender. The CTS has an additional field to store the channel ID which further degrades the performance of the proposed protocol. When the sender receives the CTS packet, it sends a RES packet (reserve) to reserve the data channel. The RES packet contains the transmission schedule and the channel ID. Overhearing nodes use the information contained in the RES and CTS packets to update their Multi-channel NAV values. The RES packet is an additional packet which also degrades the performance of the control channel.

The protocol does not provide any mechanism of ensuring that only one master node publishes control and data windows. If a number of nodes which are within the same communication zone or overlapping zones publish control and data windows schedules, more data collisions are likely to be experienced. 
In [17], a scheme employing a common default channel is implemented. The scheme relies on the services of a data structure called the Preferable Channel List (PCL) to coordinate the reservation of data channels. The reservation of data channels is done during an ATIM window on the default channel. The default channel is only implemented during the ATIM window. In the data window the default channel is used as a data channel.

The sizes of the ATIM and ATIM-ACK packets have been increased to cater for the channel information. Unfortunately, the increased packets degrade the performance of the protocol. The scheme also introduces a new packet called the ATIM-RES which also impact negatively on the performance of the protocol.

After the channel negotiation, the pair switches onto the reserved data channel to exchange the RTS and CTS packets before sending data packets. The signalling duration is too long and its overhead cost is too high. Signalling takes place on the default channel during the ATIM window and on the data channels during the data window. Furthermore, when the nodes fail to agree on one channel, data transmission is differed until the next beacon interval. Unfortunately, the nodes would have already wasted network resources.

The scheme uses the CSMA mechanism in sending the ATIM/ATIM-ACK and the RTS/CTS packets which wastes a lot of resources. During the ATIM window a data channel can be reserved by many nodes. The nodes then contend for the same data channel using the RTS/CTS packets. There is also a likelihood that a pair may fail to access a reserved data channel during the data window, which wastes bandwidth. More bandwidth is wasted during the ATIM window when all data channels are not utilized. The bandwidth is further wasted when RTS/CTS precedes a data frame on the data channels.

The implementation of a temporary control channel is also proposed in [18]. One channel is set aside as a signalling channel and it is referred to as a dedicated channel. The rest of the channels are set aside as data channels. The dedicated channel can also be used as a data channel when the contention period has ended. The protocol is divided into a contention reservation interval (CRI) and into a contention free interval (CFI). Nodes contend for network resources and data channels during the CRI. Thereafter, they all defer their transmissions until the CFI. The deferment of data transmission wastes resources and degrades both the capacity of the dedicated and data channels. The protocol also requires global synchronization, a challenge for mobile wireless nodes.

The data channels lie idle during the CFI hence their bandwidth is not utilized effectively. This is a common problem with all protocols which divides a transmission process into contention and data transmission windows.

Nodes in [19] randomly select home channels to listen on when they are idle. The proposed protocol segments a network and creates logical portions.

Each node chooses a home channel which it listens on. At start-up, nodes sense all the available channels to discover neighbours and add them to their neighbour tables. The process of updating neighbour tables is costly as nodes incur both very high channel switching and sensing costs. A very high overhead cost is also incurred when nodes switch and sense channels.

A new node can also send broadcast packets to probe neighbours. Broadcast packets flood the network and increase chances of data collisions. There is also a possibility that many nodes would not receive the broadcasted packets since they will be listening on different home channels. The responses to probing broadcast packets are also susceptible to interference and collisions.

The paper in [19] does not explain how a joining node broadcast its probing packet on one channel yet the packet is meant to be received by nodes on different channels. Before sending data, a node has to first determine a home channel of the receiver then it has to switch onto it. The node then contends for the destination node's home channel. Nodes experience longer signalling delays before they can send out their data packets. Unfortunately, the process of determining the recipient's home channel is not described in the paper. 
The paper in [20] proposes a single transceiver Multi-channel MAC protocol which implements contention and data windows. The size of the contention window is not fixed; it is adjusted using smart window increase and decrease rules. However, the smart concept is not described. A three way channel reservation handshake is implemented with an additional control packet called the Multi-channel CTS Recognition (MCTS-R). The MCTS is used by the sender to confirm the channel reservation unfortunately, it increases the signalling overhead cost. The protocol does not consider the channel switching delay. Channel switching delay cannot be avoided in a multi-channel network.

The following packets are exchanged during the control window to reserve a data channel: MRTS, MCTS and MCTS-R. The MRTS and MCTS packets are also exchanged by nodes on the data channel before they transmit data frames. There are therefore too many signalling packets that are exchanged by nodes. These packets increase the signalling overhead cost of the proposed protocol and it degrades the performance and the capacity of the control channel. There is a need to reduce the signalling overhead on the control channel to improve its scheduling capacity.

The protocol also employs the use of data structures in data channel reservation. Each node keeps the following data structures: the In-use channel list, the free channel list and busy channel list. A counter is maintained to sum up all the channels in the busy channel list, and the number of nodes which have selected them.

The data channels are not used during the control window and their bandwidth is wasted. Too many data structures are used which requires a lot of processing and storage which is a challenge in a mobile environment.

The Group Allocation Multi-hop Multiple Access (GAMMA) in [21] implements multiple transmission channels. Each transmitter has a unique channel. Nodes first join a transmission-group before they can initiate any transmission. Once the terminal has become a member of a transmission-group, it can now send all its data frames to the receiving node. The nodes do not contend for the channel and there is no interference during the transmission of data frames. Unfortunately, the proposed scheme is not connection oriented. It creates a number of logical sub networks, which may lead to a high incidence of hidden terminals. The hidden terminal problem degrades the capacity of the network as significant number of packets is retransmitted.

The GAMMA protocol divides the transmission channel into cycles. Each cycle is divided into contention and data slots. A station sends an RTS packet and receives a CTS packet during the contention slot. The RTS/CTS packets are sent when a node wishes to register with a transmission group. Once it has been registered, it is allocated a data slot to transmit all its packets. A node can also receive its data packets during its data slot. However, this approach is wasteful when a node has no data to receive and when few packets are sent.

Nodes can have different cycle lengths while their contention slots have to be aligned for nodes to exchange RTS/CTS packets successfully. The source and destination nodes require synchronization in a slot which is a challenge for wireless networks. The challenge is worsened by the difference in the transmission cycle lengths. Interestingly, if cycles are not equal, shifted or misaligned, the nodes will not be able to exchange data.

A node with a shorter cycle adds additional data slots, this waste bandwidth. Furthermore, the decision to add more data slots is communicated to the group members. The details about the increases of data slots are captured in data and ACK packets headers. All the nodes which overhear the data and ACK packets increase their transmission cycle lengths. The process of realigning data slots degrades the performance of the protocol. More bandwidth is also lost through the realignment of data slots.

Data and ACK packets also contain a flag in their headers which informs neighbouring nodes when a station is likely to reduce its cycle length. The packets headers also contain additional information on cycle lengths and cycle lengths flags. When a node has reduced its data slot size, its neighbours also reduce their data slots by the same margin for the realignment of the slots. These decisions are communicated to all neighbours which increases substantially the payload of the protocol. The proposed protocol is also complex and complicated. 
The packet header sizes may be increased to accommodate additional information. The enlarged data and ACK packets degrade the capacity of the network. The cycle information is also sent to neighbours when new nodes join a group triggering the need to resize data slots and to realign them.

A node can leave or deregister from a group if it does not have data to send or data to receive. It has to set an appropriate flag in its packet header to inform group members about its intention to deregister before it leaves the group. The membership of nodes can also be lost if a number of its data slots remain idle for a number of successive cycles. Unfortunately, significant amount of bandwidth would have been wasted already before a node with idle data slots is de-registered. The node also waste bandwidth when it registers its intention with the destination node to leave a group.

The multi-channel MAC protocols in this category split a communication session into a control and data windows. The reservation of data channel is done inside the control window through a temporary control channel and the bandwidth of the data channels is not utilized during the control window. All the data channels remain idle in the control window until the reservation of data channels has ended. The control channel is utilized as a data channel in the data window. The bandwidth which is wasted during the control window may be avoided so as to increase the capacity of the network and to improve the effectiveness of these protocols. The wasted bandwidth is caused by the MSC which needs to be solved by employing scheduling techniques which minimize the MSC.

The MSC is a dominant problem in Multi-channel MAC protocols owing to the failure of the single signalling channel to schedule simultaneously data transmissions to all the available data channels. The MSC may either be solved or minimized by improving the capacity of the control channel and its scheduling capacity.

Fig. 6 shows the functionality of multi-channel MAC protocols which implement a temporary control channel. The protocols divide a communication process into control and data windows as shown in the figure below.

\begin{tabular}{|c|c|c|c|}
\hline Reservation & Data Frame & Reservation & Data Frame \\
\hline DATA IDLE & Data Frame & DATA IDLE & Data Frame \\
\hline DATA IDLE & Data Frame & DATA IDLE & Data Frame \\
\hline
\end{tabular}

Figure 6. The poor utilization of data channels in Windows based multi-channel MAC protocols.

In Fig.6, the two windows are labelled. Each column is marked either as control window or data window. The rows represent the channels. The top row depicts the temporary control channel. In the control window, the control channel is marked "Reservation" while in the data window it is marked "Data Frame". This shows that the control channel is used for data channel reservation during the control window and then as a data channel during the data window to transmit data frames.

The last two rows depict two data channels. When data channels are being reserved during the control window, they lie idle and remain unused until the end of the control window. The bandwidth of the data channels is wasted during each and every data channel reservation phase. The protocols are very effective in utilizing the channels during the data window.

Figure 7 depicts a strategy which addresses the MSC in the control channel. Unfortunately, the challenge remains unsolved in the data channels. The technique utilizes the control channel during the control and the data windows. The control channel is utilized for both data reservation during the control window and for data transmission during the data window. The strategy which addresses the MSC in both the control and data channel is therefore sought after. However, Figure 7 demonstrates that such a solution is not farfetched. 
The elimination or reduction of the idle periods of data channels which is repetitive can solve the MSC and avail more bandwidth. The scheduling capacity of the control channel can also be improved when the MSC is reduced.

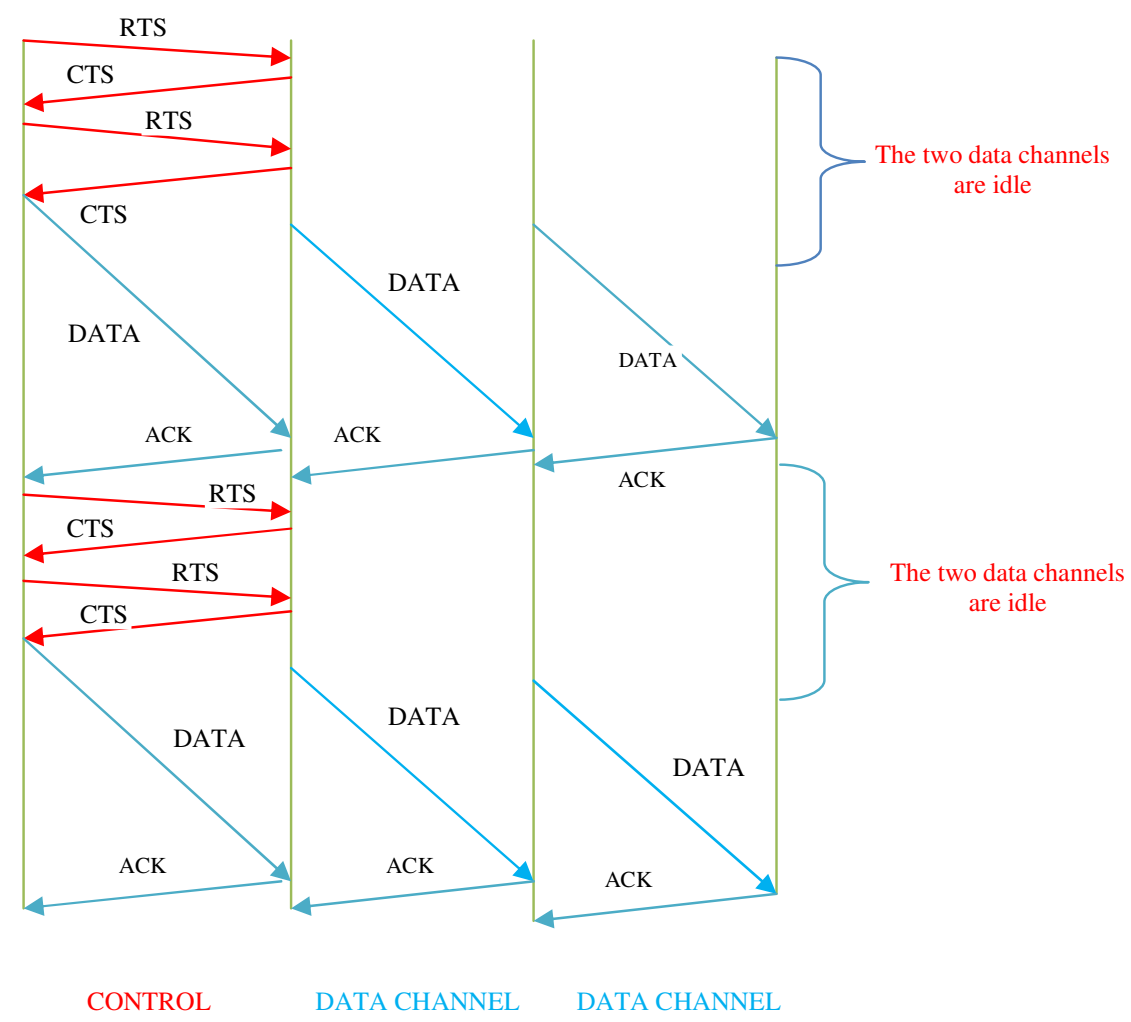

Figure 7. The underutilization of data channels

\section{Dedicated Control Channel MUlti-Channel MAC protocols}

The multi-channel MAC protocols in this category employ a single radio and one dedicated control channel. The control channel is used exclusively for signalling purposes while the rest of the channels are earmarked for data transmission. The protocols also suffer from the MSC and the poor utilization of channels. However, the MAC protocols in this category do facilitate network connectivity. They provide nodes with a common reference point, the dedicated control channel on which nodes listen on when they are idle. The control channel therefore helps in the synchronization of nodes.

The control channel is the driver of the multi-channel MAC protocols. It can either improve the performance of the MAC protocols or degrade them depending on its design. If the control channel has limited capacity it may cause a system bottleneck. On the hand if it is well designed and has enough capacity it can improve the performance of the network. The signalling overhead therefore may be reduced to improve the capacity of the control channel. We review the effectiveness of the MAC protocols in reducing the signalling overhead. It is envisioned that the reduction of the signalling overhead improves the performance of the MAC protocols which implement a dedicated control channel.

The protocol in [22] introduces the idea of distributed information sharing to ensure that communicating pairs do not make independent decisions. Neighbouring nodes can notify a transmitter-receiver when they perceive a conflict. Unfortunately, there is no effective solution given to solve collisions caused by simultaneous notifications. The neighbouring nodes can also notify the pair of the missing nodes so that they can defer their transmissions to deaf nodes. 
The proposed protocol does implement a dedicated control channel however; it is optimized to solve the terminal deafness problem and collisions on data channels. The paper also analyzed the single control channel bottleneck problem and concluded that the problem can be predicted and avoided. The channels are assumed to be orthogonal. The protocol introduces up to six control packets which are exchanged on the control channel before a data channel is reserved. There is a lot of signalling involved causing a high overhead costs.

The signalling overhead degrades significantly the capacity and the scheduling capacity of the control channel. The signalling overhead may be reduced to improve the capacity of the control channel. Furthermore, the MSC is repetitive and may be addressed to improve the performance of the protocol.

A Distributed Queue Dual Channel (DQDC) scheme is proposed in [23]. The scheme seeks to increase the utilization of data channel and to increase the achievable throughput. The scheme uses one control channel and at least one data channel. The control packets are transmitted on the control channel. The control packets are exchanged by nodes on the control channel which wish to reserve one of the available data channels. Data frames and ACK packets are transmitted on the data channels.

Nodes maintain a distributed queue (DQ) and update their reservation DQ regularly to avoid collisions on the data channel. To update the reservation DQ the node relies on the information contained in the overheard control packets. The scheme maintains a distributed queue of all communicating pairs which have reserved the data channels. The DQDC introduces a four way packet handshake negotiation scheme. The following packets are exchanged before a data channel is reserved: Mesh Transmission Opportunity Request (MTXOP REQ), Mesh Transmission Opportunity Response (MTXOP RSP), Mesh Transmission Opportunity Acknowledgment (MTXOP ACK) and Agreement Indicator (AID).

The sender first sends a MTXOP REQ which includes the IDs for the sender and receiver. It also contains the duration and starting time of the transmission. If the receiver rejects the sender's schedule it responds with the MTXOP RSP packet and includes its own preferred transmission schedule indicating its duration and its starting time of the transmission. The sender then sends back the MTXOP ACK packet accepting the receiver's preferred transmission schedule. The MTXOP ACK can be sent when a given node is either rejecting or accepting a proposed transmission schedule. The MTXOP ACK is sent in reply to either the MTXOP REQ or the MTXOP RSP. The receiver responds with the AID packet to complete the data channel reservation. The AID is sent by the node which has received the MTXOP ACK. It is broadcasted to all the nodes to advertise the transmission agreement reached by the sender and receiver. It marks the conclusion of the negotiation process.

The signalling overheard is too high and it degrades the capacity of the control channel. It also increases the effects of the MSC. However, a three way handshake is possible where the receiver accepts the sender's MTXOP REQ. The receiver will send back the MTXOP ACK if it accepts the sender's preferred transmission schedule. Thereafter, the sender will broadcast the AID to advertise the agreement. Though a three way handshake has less impact on the control channel and MSC, it is still too high. There is a need to reduce the signalling overheard in both cases to improve the efficiency of the control channel. One other possible way is to equip the network with intelligence to aid in the reservation of data channels to avoid long control channel handshakes.

When the data channel has been reserved neighbouring nodes are notified through the AID which is sent by the node receiving the MTXOP ACK packet. The AID is a broadcasted packet which fails to reach nodes which are currently transmitting on the data channels and those which are hidden to the AID broadcasting node. As a result, the nodes with insufficient knowledge of the network status may cause data collisions and retransmissions. The retransmission of packets further degrades the protocol.

Nodes returning to the control channel assume that the data channels are busy until they overhear one of the control packets or after the expiry of the set threshold. If the returning node has a data to transmit, it has to defer its transmission affecting the quality of service provisioning of time bounded data packets. Furthermore the signalling overhead is significant and it degrades the performance of the protocol. Nodes have to update their DQ each time they receive an AID or the MTXOP ACK packet. The DQ requires a node with unlimited processing power. Unfortunately mobile nodes suffer from both low capacity and limited storage and processing power. 
A scheme implementing a separate control channel and $\mathrm{N}$ traffic channels is proposed in [24]. The authors attest that the number of channels should be less than the number of terminals for the efficiency of the protocol. A node can transmit and receive on any of the available traffic channels. The RTS/CTS packets are sent on the control channel and a data channel is reserved by the receiver. When the receiver receives an RTS, it selects the clearest channel to be reserved and then sends the CTS packet. Unfortunately, a CTS based data channel reservation scheme fails to calm hidden nodes at the sender's neighbourhood. Data packets can be destroyed by hidden nodes at data reception forcing the protocol to retransmit the affected packets. Retransmissions do degrade the performance of the control channel.

A node first senses all the data channels including the control channel before it sends an RTS packet. It then embeds the list of all free data channels in the RTS packet. This means that the RTS's length is increased to accommodate the channel list information to be sent to the receiver. Upon receiving the RTS, the receiving node first senses all the channels and then it compares its channel list with the one sent by the sender. The destination node then identifies common free channels and selects the best from the list. The destination node then sends the CTS packet to the source node embedding the best data channel. The scheme does not consider channel switching penalty in its timeouts. When the destination node fails to select a data channel, it does not send back the CTS packet. The source node will have to try after the timeout of the CTS packet. This is a good approach which ensures that the control channel is not degraded with reattempts and retransmissions however; the scheme suffers from high sensing related costs.

Nodes are assumed to be able to carrier sense all the channels simultaneously. However, this is not possible. Nodes can sense one channel at a time and then switch onto the next channel before sensing it. This forces a node to incur high overhead costs which relate to sensing and channel switching delays. It is further assumed that a node can receive multiple packets on different channel simultaneously which is not possible with a single radio.

The data channels are idle during the contention phase; hence their bandwidth is not utilized effectively. This challenge is common with all the multi-channel MAC protocols and it needs to be addressed to improve the efficiency of multi-channel MAC protocols.

In [25], an asynchronous Multi-Channel Coordination Protocol (AMCP) is proposed. The protocol employs a single transceiver and a dedicated common control channel. Nodes contend for the control channel using the RTS/CTS packets and then reserve the data channels using channel table information. An RTS packet is send with a list of free data channels and a preferred data channel. If the preferred data channel is free at the receiver a confirming CTS packet is sent back to the sender, otherwise a rejecting CTS packet is sent. Data channels timers are stored in the local channel tables and the respective data channels are reserved when their timers expire.

When the sender's preferred data channel is rejected by the receiver, the sender has to randomly select the receiver's suggested data channels and restarts the contention cycle. In a worst case scenario the repeated contention cycles may increase exponentially resulting in long signalling delays, degrading significantly the capacity of the control channel.

The protocol may work well when the network is assumed be in operational, unfortunately it may fail when it is implemented in a newly deployed network. All nodes will set all the data channels unavailable and enter into an indefinite wait for control packets. All the nodes will defer their transmissions and there will be no control packets that would be overheard on the control channel. As a result, the nodes will fail to update their local channel tables and schedule their next transmissions.

A similar challenge can be encountered by joining nodes. A joining node has to first set all the channels unavailable and defer its next transmission until it overhears control packets. It then updates its local channel table before contending for the control channel. Assuming that all the nodes which are on the network have no data to send, the new node will not be able to send its packets until the resident nodes start communicating. In this case the new nodes will be blocked due to the lack of adequate knowledge of the status of the network.

The challenge of newly deployed networks and of the nodes which join an inactive network can be solved by allowing nodes to sense the data channels before initiating a communication. Unfortunately, sensing all the channels increases substantially the signalling overhead which in turn degrades the capacity of the 
control channel. The nodes have to sense and switch between channels, which is a heavy penalty to pay. A network supported contention scheme may be a cheaper and effective solution.

The rejecting CTS packet which is sent back to the sender by the AMCP protocol includes a list of data channels which are free to inform the sender about the available data channels. The sender will select one of the listed data channels in its next attempt. Unfortunately the CTS packet does not include timers of the unavailable data channels to help a sender which would want to contend for the currently unavailable data channel when it becomes free.

It is possible that all the data channels may be busy at the receiver and the timer information may be of vital importance if it were included in the rejecting CTS. The free data channels can be reserved by other nodes ahead of a given node. Furthermore, successive new contention cycles waste the capacity of the control channel and increases its saturation rate.

It should also be noted that the sizes of both RTS and CTS packets may be increased to cater for additional information to be sent. The rejecting CTS packet is likely to be a couple of bytes larger than the standard CTS. This means than more bandwidth will be required to transmit the new RTS, the Confirming CTS and the rejecting CTS packets. Given the need for more capacity of the control channel, any further degradation of the control channel may be avoided.

There is also a need for local channel tables to be stored and to be updated frequently. However, nodes have limited storage and processing power. The ACMP is also affected by the MSC and its effect is repetitive. The multi-channel MAC protocols should endeavour to improve the capacity of the control channel and reduce both the signalling overhead costs and the effects of the MSC.

In [26], a scheme employing busy signals is proposed. The scheme assumes that a transceiver can listen on all the channels simultaneously. A single channel is divided into two sub channels, a control and a data channel. The control channel is used to send the control packets while the data frames are sent on the data channel. While the nodes are sending and receiving data they transmit busy tones on the control channel. During the transmission of data frames, the control is not used. It transmits busy tone signals. This wastes the capacity of the control channel and reduces its scheduling capacity. The data channels are also not used during data channel reservation while the control channel is underutilized during data transmission. The well coordinated use of the channels solves the MSC and to improve the efficiency of the protocol. The scheme assumes that a node can send and listen at the same time, and also that a node can transmit on two channels simultaneously, which is not possible.

\section{Channel Hopping Multi-Channel MAC protocols}

In this section, we analyze multi-channel MAC protocols which do not implement a control channel. The protocols implement a channel or frequency hopping concept. The coordination of channels and the synchronization of hoping sequences is examined. The channel hoping technique though a good idea, it is complex and does not provide a common reference for the terminals to synchronize. This leads to the partitioning of the network and the creation of logical segments in the network.

The Slotted Seeded Channel Hopping ( $\mathrm{SSCH}$ ) [27] is a time based protocol and its performance is affected by a need for global time synchronization. The SSCH suffer from the MRP and hidden terminal problem. If the receiver is deaf, the sending node will be allowed two attempts in one slot. The allocation of two slots to one node wastes bandwidth and causes signalling delay. FIFO queues are maintained at each node and packets are transmitted in a round robin basis in a given neighbourhood. Packets which are not transmitted due to MRP, their priorities are reduced. However, it is not clear how the nodes are grouped into different neighbourhoods. The head of line (HOL) problem is also a challenge in this scheme. A packet in the head of the queue cannot be removed to make way to the next packet with a higher priority. When a number of packets which are destined to one node are not delivered after the expiry of the slot, they are all dropped after wasting a significant amount of bandwidth and network resources.

A node switches to all the available channels in each schedule. Given the maximum channel switching delay of $224 \mu$ s, the switching overheard is too high and heavy. The notion of slotted and cyclical data 
transmission is good. However, nodes should be limited to very few channels in any given slot to reduce signalling delay. Furthermore, the SSCH requires clock synchronization to ensure that slots of different nodes start and end at the same time. Nodes are expected to send, receive or forward packets during a given slot. This means that technically, there are receiving, sending and forwarding slots and during these slots some data packets may be buffered. For example, if one node is going to be sending its packets in the next slot, all its coming packets will be buffered. This causes longer transmission delays which may be reduced to improve the performance of the network. The delay sensitive packets are degraded when they are buffered.

The scheme proposed in [28] is a frequency hopping scheme in which radios hop between available channels. The scheme implements polling and it wastes bandwidth when a polled node does not have data to send. It is not robust and flexible. The protocol requires synchronization which is a challenge in mobile networks. Furthermore, the receiver reserves the channel and fails to notify nodes which are in the carrier sensing zone of the sender and which are hidden to it.

The idea of a common channel is also exploited. Nodes listen on the common channel, in an attempt to synchronize their hopping sequences. Carrier sensing is not implemented, and nodes rely on their hopping sequences.

When the nodes begin their hoping sequences, they first enter into PASSIVE state which is equal to the time spent on a hop (dwell time), and then they move into a ready-to-receive (RTR) state on each hop before they transmit data. During the RTR state, an RTR packet is sent to the target destination. The source responds with data packets for the polling node in the XMIT state, otherwise it responds with a CTS packet. When the CTS packet is sent, signalling duration increases. Furthermore, during the RTR state nodes send the RTR packets without sensing the current frequency hop which may result in RTR collisions.

W-CHAMB proposed in [29] is a TDMA based scheme which requires global clock synchronization. The protocol implements two in-band energy signals which waste bandwidth. The W-CHAMB implements the idea of prioritized access. The concept has three phases; these are the Prioritization Phase (PP), Contention Phase (CP), and the Transmission Phase (PP). There is a lot of contention which takes place during the PP and the $\mathrm{CP}$ result in high overhead costs. Data frames are assigned priority levels which are used to prioritize the transmission of data packets. However, it is not clear how the scheme solves the HOL problem when a packet with the highest priority is blocked by the one with less priority.

The protocol proposed in [30] is called the Multi-channel MAC (McMAC). Nodes hop in a pseudo random fashion, independent of each other. A node is expected to synchronize with its neighbour's hopping sequence. Unfortunately, synchronization is not possible in wireless communications.

To address the synchronization problem, a guard time is implemented by the McMAC protocol to ensure that neighbourhood nodes are synchronized. The idea of the guard time is good; unfortunately, it further degrades the performance of the protocol. The bandwidth used as a guard band can be used for data transmission purposes. Every packet sent includes a 32 bit field which stores current time and seed. The time stamp increases the payload of the protocol.

A new node which joins a network has to first wait for ten seconds before it establishes and follows its hoping sequence. On the other hand courtesy HELLO packets are sent to a newly discovered neighbour. The HELLO messages and the ten seconds waiting period do degrade the performance of the proposed protocol.

\section{OPEN RESEARCH ISSUES}

A handful of open research issues and gaps still exist in the multi-channel MAC protocols which are designed for wireless access networks such as the wireless mesh networks. For example, to date there is no paper which has attempted to address the MSC. The existing MAC protocols which implement either a dedicated or temporary control channel do under utilize the bandwidth of the data channels during the reservation phase. The control channel is not able to schedule successful data transmission to all data 
channels simultaneously. As a result the bandwidth of the idle data channels is wasted. This problem is cyclical and it does degrade the performance of MAC protocols.

The MSC can be addressed in twofold. It can either be reduced or limited to the first cycle. Therefore, there is a need to address the MSC for better performance.

A number of multi-channel MAC protocols have either introduced a few new control packets or increased the sizes of the existing control packets. Though the techniques are designed to reduce the channel sensing and channel switching penalty in conjunction with the use of the data structures, they increase significantly the signalling overhead. The signalling overhead degrades the capacity of the control channel and impact negatively on its scheduling capacity.

Design techniques which reduce the control channel signalling overhead should be designed. The main goal of the multi-channel MAC protocol implementing a control channel may minimize signalling overhead. The capacity of the control channel may be increased for better performance to be realized.

Multi-channel MAC protocol designers assume that all the channels are orthogonal. Unfortunately, the channels do overlap. If the channel guard bands were to be increased to ensure orthogonality of channels, very few non overlapping channels would be created. Implementing a control channel in a system with very few channels does not offer any performance benefits. A number of good MAC protocols which have been designed will not serve their intended purposes if the channels were few. There is a need to closely examine this issue. Possible strategies of increasing the number of non overlapping channels should be investigated. The use of CDMA may be closely investigated in this regard for its interference averaging and resisting properties.

Lastly, the optimum number of channels that can be supported by a single radio before any need to use multi radios may be considered and investigated. Increasing the number of radios without taking into consideration the efficiency and optimality of the multi-channel MAC protocols may prove to be every expensive. The envisioned performance of such multi radio systems may not justify a need for such expensive and complex design approaches.

\section{CONCLuSION}

The wireless mesh networks are a promising candidate for the fourth generation networks (4GN). However, the limited capacity of the WMN may be addressed to meet the envisioned 4GNs high data rates. The MAC protocol designers are currently researching on strategies that may avail more capacity. One of the active research areas in this regard is the implementation of multi-channel MAC protocols.

The multi-channel MAC protocols which implement a control are promising in the sense that they facilitate network connectivity and provide nodes with a common reference point. Nodes listen on the control channel and can quickly synchronize on it. The control channel approach requires optimization and to be enhanced. The underutilization of data channels during contention should be also addressed.

The number of available orthogonal channels may be closely investigated. On the other hand, new techniques of reducing interference between overlapping channels may be considered. The implementation of CDMA could be one of the possible approaches which can avail more orthogonal channels. Lastly, good coordination and channel scheduling techniques can also address these challenges.

\section{REFERENCES}

[1] S.-L.Wu, C.-Y. Lin, Y.-C. Tseng, and J.-P. Sheu. New Multi-Channel MAC Protocol with OnDemand Channel Assignment for Mobile Ad Hoc Networks. In Proc. International Symposium on Parallel Architectures, Algorithms and Networks (I-SPAN), Dallas / Richardson, TX, USA, December 2000 .

[2] Ian F. Akyildiz, Xudong Wang, and Weilin Wang (2004). Wireless mesh networks: a survey. Computer Networks, www.elsevier.com/locate/comnet 
[3] The editors of IEEE 802.11. Wireless LAN Medium Access Control (MAC) and Physical Layer (PHY) Speci_cation, 1997.

[4] Shih-Lin Wu, Yu-Chee Tseng, Chih-Yu Lin and Jang-Ping Sheu (2002). A multi-channel MAC protocols with power control for multi-hop mobile ad hoc networks. The computer journal, Vol. 45, No. 1, 2002.

[5] Chandrakanth Chereddi, Pradeep Kyasanur, and Nitin H. Vaidya Design and Implementation of a Multi-Channel Multi-Interface Network, REALMAN'06, May 26, 2006, Florence, Italy

[6] Chandrakanth Chereddi. SYSTEM ARCHITECTURE for multichannel multi-interface wireless networks, University of Illinois at Urbana-Champaign, 2006

[7] P. Kyasanur and N. H. Vaidya. Routing and Link-layer Protocols for Multi-Channel Multi-Interface Ad hoc Wireless Networks. Mobile Computing and Communications Review, 10(1):31-43, Jan 2006.

[8] Marco Di Felice, Guomei Zhu and Luciano Bononi (2008). Future Channel Reservation Medium Access Control (FCR-MAC) Protocol for Multi-Radio Multi-Channel Wireless Mesh Networks PEWASUN'08, October 27-28, 2008, Vancouver, BC, Canada.

[9] Hon Sun Chiu, Kwan L. Yeung, and King-Shan Lui (2009). J-CAR: An Efficient Joint Channel Assignment and Routing Protocol for IEEE 802.11-Based Multi-Channel Multi-Interface Mobile Ad Hoc Networks. IEEE TRANSACTIONS ON WIRELESS COMMUNICATIONS, VOL. 8, NO. 4, APRIL 2009

[10] Junaid Qadir, Chun Tung Chou, Archan Misra, and Joo Ghee Lim (2009). Minimum Latency Broadcasting in Multiradio, Multichannel, Multirate Wireless Meshes. IEEE TRANSACTIONS ON MOBILE COMPUTING, VOL. 8, NO. 11, NOVEMBER 2009

[11] Peng-Jung Wu and Chung-Nan Lee (2009). Connection-oriented multi-channel MAC protocol for adhoc networks. Computer Communications 32 (2009) www.elsevier.com/locate/comcom

[12] Rongsheng Huang, Hongqiang Zhai, Chi Zhang, and Yuguang Fang (2008). SAM-MAC: An efficient channel assignment scheme for multi-channel ad hoc networks, Computer Networks 52 (2008). www.elsevier.com/locate/comnet

[13] Ashish Raniwala, Kartik Gopalan and Tzi-cker Chiueh. Centralized Channel Assignment and Routing Algorithms for Multi-Channel Wireless Mesh Networks, Mobile Computing and Communications Review, Volume 8, Number 2

[14] K Pradeep and N. H. Vaidya, "Routing and link-layer protocols for multi-channel multi-interface ad hoc wireless networks," ACM SIGMOBILE Mobile Computingand Communications Review, Vol. 10, 2006, pp. 31-43.

[15] CHI-YU LI, AN-KAI JENG AND RONG-HONG JAN. A MAC Protocol for Multi-Channel MultiInterface Wireless Mesh Network using Hybrid Channel Assignment Scheme. JOURNAL OF INFORMATION SCIENCE AND ENGINEERING 23, 1041-1055 (2007)

[16] Ritesh Maheshwari, Himanshu Gupta and Samir R. Das. Multichannel MAC protocols for wireless networks. SECON 2006, Volume 2, Issue , 28-28 Sept. 2006.

[17] Jungmin So and Nitin vaidya (2004). Multi-Channel MAC for Ad Hoc Networks: Handling MultiChannel Hidden Terminals Using A Single Transceiver. MobiHoc 2004, Roppongi, Japan, ACM.

[18] Jenhui Chen, Shiann-Ysong Sheu. Distributed Multichannel MAC protocol for IEEE 802.11 ad hoc wireless LANs. Computer Communications (2005). www.elsevier.com/locate/comcom

[19] Hoi-Sheung Wilson So and Jean Walrand (2006). Design of a Multi-Channel Medium Access Control Protocol for Ad-Hoc Wireless Networks. University of California at Berkeley

[20] Wen-Tsuen Chen, Jen-Chu Liu, Ting-Kai Huang, and Yu-Chu Chang (2008). TAMMAC: An Adaptive Multi-Channel MAC Protocol for MANETs. IEEE TRANSACTIONS ON WIRELESS COMMUNICATIONS, VOL. 7, NO. 11, NOVEMBER 2008

[21] Andrew Muir and J.J. Garcia-Luna Aceves. A channel access protocol for multi hop wireless networks with multiple channels. University of California.

[22] Tie Luo, Mehul Motani, and Vikram Srinivasan (2009). Cooperative Asynchronous Multichannel MAC: Design, Analysis, and Implementation. IEEE TRANSACTIONS ON MOBILE COMPUTING, VOL. 8, NO. 3, MARCH 2009

[23] Ali Khayatzadeh Mahani, Majid Naderi, Claudio Casetti, and Carla F. Chiasserini (2007). ENHANCING CHANNEL UTILIZATION IN MESH NETWORKS. IEEE MILCOM, Orlando, FL, 2007, 29-31 October 2007. www.ieexplore.ieee.org/xpls/abs_all.jsp?arnumber $=4454826$

[24] Jain Nitin, Samir R. Das and Asis Nasipuri. A multichannel CSMA MAC protocol with ReceiverBased Channel Selection for Multihop Wireless Networks. 
International Journal on AdHoc Networking Systems (IJANS) Vol. 4, No. 1, January 2014

[25] Jingpu Shi, Theodoros Salonidis, and Edward W. Knightly (2006). Starvation Mitigation Through Multi-Channel Coordination in CSMA Multi-hop Wireless Networks, MobiHoc'06, May 22-25, 2006, Florence, Italy

[26] Rui Zhao and Bernhard Walke (2005). Decentrally controlled wireless multi hop mesh networks for high quality multi media communications. MSWiM October 2005. http://portal.acm.org/citation.cfm?id=1089444.1089479 6 June 2007

[27] Paramvir Bahl, Ranveer Chandra and John Dunagan (2004). SSCH: Slotted Seeded Channel Hopping for Capacity Improvement in IEEE 802.11 Ad-Hoc Wireless Networks, MobiCom' 04, Sept. 26-Oct. 1, 2004, Philadelphia, Pennsylvania, USA.

[28] Asimarkis Tzamalonkas and J.J. Garcia-Luna-Aceves. A receiver-Initiated Collision-Avoidance Protocol for Multichannel Networks. www.cse.ucsc.edu/ccrg/.../jamal.infocom01.pdf, IEEE Infocom 2001

[29] J. Deng and Z. Haas (1998). Dual Busy Tone Multiple Access (DBTMA): A New Medium Access Control for Packet Radio Networks, in Proc. of IEEE ICUPC, Florence, Italy, 1998.Hoi-Sheung Wilson So, Jean Walrand and Jeonghoon Mo. McMAC: A Parallel Rendezvous Multi-Channel MAC Protocol, www.walrandpc.eecs.berkeley.edu/Papers/McMAC.pdf 